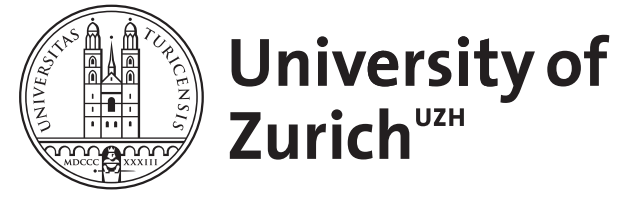

\title{
Heidnisches Können in christlicher Kunst
}

Schnyder, Mireille

Posted at the Zurich Open Repository and Archive, University of Zurich ZORA URL: https://doi.org/10.5167/uzh-94063

Book Section

Published Version

Originally published at:

Schnyder, Mireille (2014). Heidnisches Können in christlicher Kunst. In: Köbele, Susanne. Literarische Säkularisierung im Mittelalter. Berlin: Walter de Gruyter, n/a. 


\section{Mireille Schnyder}

\section{Heidnisches Können in christlicher Kunst}

\section{Schein und Sein}

In Mandevilles Reisen (3. Viertel 14. Jh.), diesem kompilierten Reisebericht eines belesenen Stubenhockers, der aber über Jahrhunderte in alle möglichen Sprachen übersetzt als tatsächlicher Reisebericht die europäischen Fremdbilder prägte, findet sich - zwischen sehr viel spektakuläreren Darstellungen östlicher Regionen - die kurze Beschreibung der Insel Dracorde. Auf dieser Insel lebt ein Volk, das mit allen Attributen fehlender Kultur und Zivilisation belegt wird: Es baut weder Wein noch Korn an, lebt mehr oder weniger nackt in Höhlen, hat keine artikulierte Sprache und ernährt sich von Schlangen. Dabei verehrt, begehrt und liebt es als größten Schatz einen vielfarbigen Stein, nur wegen seiner farbigen Schönheit. Von irgendwelchen Kräften im Stein wissen die Inselbewohner nichts, wie explizit gesagt wird:

Zi̊ dracorde sind die lüte wild vnd hand tieren sitten und hand nit husser vnd wonend in hülinen vnd redent nit. Aber sie huwlent vnd plerent so sie zornig oder wol gemuit sind vnd buwent weder win noch korn vnd essent nit anders denn schlangen. Vnd der ist gni̊g da vnd begerent kains anderen richtüms denn eins steins. Der da zů land wachset vnd heisset darcordica vnd ist der selb stein ir rihtumz vnd ir schatz vnd wissend doch nit was krafft er hat. Vnd hat der selb stein wol sechtzigerley farben vnd so menig krafft. ${ }^{1}$

Mehr als deutlich wird hier die Lust am sinn- und zwecklosen schönen Schein einem unkultivierten Volk zugeschrieben. Dabei wird die Reaktion der Inselbewohner auf die Schönheit als Begehren bezeichnet; in der Übersetzung von Velser heißt es, dass sie nicht wissen, wozu dieser Stein gut sein soll, außer das sy in lieb haben von seiner

1 Jean de Mandeville, Reisen. Reprint der Erstdrucke der deutschen Übersetzung des Michel Velser (Augsburg, bei Anton Sorg, 1480) und des Otto von Diemeringen (Basel, bei Bernhard Richel, 1480/81). Hrsg. und mit einer Einleitung versehen von Ernst Bremer, Klaus Ridder. Hildesheim/Zürich/New York 1991 (Deutsche Volksbücher in Faksimiledrucken Reihe A. 21), hier: Diemeringen (1480/81), S. 306 f. 
schoone wegen. ${ }^{2}$ Dieses sinnlose Begehren des schönen Scheins ist im christlichen Fremdheitsdiskurs Grundkonstituens einer fremden, nicht-christlichen und damit auch falschen Begehrensstruktur. Es ist aber auch diese sinnleere Faszination durch äußerliche Form und Glanz des Materials, die zu den Grundargumenten christlicher KunstKritik und Kunst-Skepsis gehört.

Weist die Verehrung des schönen Steins durch die Dracordier aber lediglich auf deren vollkommene Unwissenheit hin, entsteht die wirkliche Gefahr für den Menschen über die kunstvolle Zurichtung der kostbaren Materialien, die zweite Schöpfung und Nachahmung der creatura. ${ }^{3}$ Das unkultivierte, ignorante und so auch noch weitgehend unschuldige Begehren nach dem schönen Schein des Schöpfungswerks wird durch das kunstvolle mimetische Bildwerk gesteigert zu einem Begehren des falschen, das heißt aber immer auch teuflisch infizierten Scheins. Denn das menschengemachte Bild ist Mittel, eine falsche Präsenz vorzutäuschen, Illusionen zu erzeugen und den Verstand auszuschalten. ${ }^{4}$

Und ist - aus christlicher Perspektive - die Verehrung des schönen Steins insofern defizient, als sie den Kern der Erscheinung nicht erkennen kann, weder die dem Stein innewohnenden Naturkräfte, noch die Schöpferhand dahinter, ist die Verehrung des von Künstlerhand in täuschender Nachahmung gemachten Bildes die Unfähigkeit, Zeichen zu lesen, die präsentische Illusion auf eine über das Zeichen zu erschließende Bedeutung zu durchbrechen, das Bild als Zeichen zu erkennen und als präsentische Realität zu destruieren. Es ist diese Unfähigkeit, die - im christlichen Erzählrahmen - die Bühne bereitet, auf der sich in täuschender Nachahmung und im Vergessen der Materialität und Technizität, das heißt der Bedingungen der Kunst, der Teufel inszeniert. ${ }^{5}$

2 Michel Velser (1480) (Anm. 1), S. 124. Vgl. auch: Sir John Mandevilles Reisebeschreibung in deutscher Übersetzung von Michel Velser. Nach der Stuttgarter Papierhandschrift Cod. HB V 86. Hrsg. von Eric John Morrall. Berlin 1974 (Deutsche Texte des Mittelalters. 66), S. 121, Z. 7-14.

3 Dies zeigt sich schon in der im Mittelalter gut bekannten Ursprungsgeschichte des Götzenbildes, der Geschichte des König Ninus. Vgl. dazu: Mireille Schnyder: Die Verfügbarkeit des Teufels und die Kunst. In: Paragrana 21 (2012): UnVerfügbarkeit. Hrsg. von Ingrid Kasten. Berlin 2012, S. 47 59.

4 Seit den frühen christlichen Schriften wird dies immer wieder betont. Vgl. u. a. Minucius Felix, der in seinem „Octavius“ den Christen sagen lässt, dass „,der Verstand der Unwissenden getäuscht ist durch die Perfektion der Kunst, geblendet durch das Glänzen des Goldes, verdunkelt durch das Leuchten des Silbers und die Weisse des Elfenbeins." Marcus Minucius Felix: Octavius. Lateinisch/Deutsch. Hrsg., übersetzt und eingeleitet von Bernhard Kytzler. München 1965, S. 136 f. (Kap. 24). Vgl. zu der Diskussion der Materialität von Bildern und deren Bewertung im westlichen Mittelalter auch mit einschlägigen Belegen Caroline Walker Bynum: Christian Materiality. An Essay on Religion in Late Medieval Europe. New York 2011, S. 46-52 u. ö.

5 In der gleichen Logik wird topisch den nicht-christlichen Religionen die Fähigkeit der Exegese mit mehrfachem Schriftsinn, die allegorische Lektüre, abgesprochen. Vgl. zu dieser, im religionspolitischen Diskurs bis heute wirksamen Topik die immer wieder anzutreffende Behauptung, dass der Koran rein literal zu lesen sei. 
Das nun aber stellt das christliche Kultbild in einen gefährlichen Kontext, von dem es aufwändig und mit immer neuen rhetorischen und inszenatorischen Tricks distanziert werden soll. Das wird entweder dadurch versucht, dass das Kultbild dem Bereich der menschlichen Künste entzogen wird, oder dass der Zeichencharakter des Bildes ausgestellt wird. Im ersten Fall wird dem Bild, als einem nicht von Menschenhand geformten (non manu factum), jede Möglichkeit einer menschlichen, intentionalen, das heißt auch willkürlichen Manipulation der Bildwirkung entzogen und ein direktes Wirken Gottes über das Bild konzipiert. ${ }^{6}$ Zur intrikaten Abgrenzung vom teuflisch gewirkten Bild, das topisch über den Hinweis auf seine Materialität und Leblosigkeit destruiert wird, werden die Entstehung des Bildes und seine technische Beschaffenheit konsequent ausgeklammert und verwischt. ${ }^{7}$ Der zweite Fall dagegen zielt auf eine intellektuelle Brechung der Rezeption des Bildes in eine Reflexion seiner Bedeutungsstrukturen und eine die (oberflächliche) Zeichenhaftigkeit und Materialität übersteigende Erkenntnis. ${ }^{8}$ Der christliche Kunstdiskurs interessiert sich entsprechend auffallend nicht für die Kunstproduktion, sondern fast ausschließlich für die Kunstrezeption. Christliche Bildkritik ist Rezeptions- und Wahrnehmungskritik.

Die Kunstfertigkeit als handwerkliches Geschick, als Beherrschung von Materie und konzeptuelle Kraft im Sinne einer mimetischen Erschließung natürlicher Potenzen, interessiert nicht. ${ }^{9}$ Denn dieses, auf ein artes-Wissen sich gründende Können, wird im heilsgeschichtlichen Narrativ in eine Tradition gestellt, die dem Geschlecht von Kain und dem verfluchten Noah-Sohn Cham zugeschrieben wird, zu deren Linie dann auch der Erfinder der Magie, Zoroaster, gehört. ${ }^{10}$ Angelegt auf eine bewusste Sinnestäu-

${ }^{6}$ Vgl. dazu Hans Belting: Bild und Kult. Eine Geschichte des Bildes vor dem Zeitalter der Kunst. München 1990, S. 95. Die kleinen anbetenden Stifter-Figuren, die schon in den frühen Heiligenbildern mit inszeniert wurden, indizieren genau diese Charakteristik des Bildes und konstituieren über die Geste der Anbetung die spezifische Wirkmacht des Bildes. Vgl. ebd., S. 93.

7 Vgl. Belting (Anm. 6), S. 65 f. Dabei wird auf eine Argumentation zurückgegriffen, die schon aus dem Bild-Diskurs der Antike bekannt ist: Das Bild soll nicht von Hand gemacht sein (non manu factum). Vgl. dazu Thomas Pékary: Imago res mortua est. Untersuchungen zur Ablehnung der bildenden Künste in der Antike. Stuttgart 2002 (Heidelberger Althistorische Beiträge und Epigraphische Studien. 38).

${ }^{8}$ Vgl. z. B. die seit dem 11. Jh. nachweisbaren Rahmeninschriften bei Passions- und Heiligendarstellungen: Nec deus est, nec homo, praesens, quam cernis imago // Sed Deus est, et homo, quem sacra figurat imago. Vgl. dazu Ragne Bugge: Effigiem Christi, qui transis, semper honora. Verses Condemning the Cult of Sacred Images in Art and Literature. In: Acta ad archaeologiam et artium historiam pertinentia 6 (1975), S. 127-139.

9 Zum Verständnis von Naturähnlichkeit und Naturnachahmung vgl. Andreas Speer: Kunst und Schönheit. Kritische Überlegungen zur mittelalterlichen Ästhetik. In: Scientia et ars im Hoch- und Spätmittelalter. Hrsg. von Ingrid Craemer-Ruegenberg, Andreas Speer. Berlin/New York 1994 (Miscellanea Mediaevalia. 22), S. 945-966, hier v. a. S. 952-954.

10 Vgl. dazu mit entsprechenden Literatur- und Quellenangaben Schnyder (Anm. 3), S. 48, Anm. 3.; S. 53, Anm. 17 und 18. Künstlerschaft im Sinne von handwerklichem und technischem Geschick wird so in den Kontext der Magie und Zauberei gestellt. Vgl. Udo Friedrich: Contra naturam. Mit- 
schung, eine innerweltliche und menschengemachte Scheinwelt, geleitet von Interessen der Repräsentation, der Macht, der Unterhaltung, der Wissensvermittlung, der Verzierung und der scheinbaren Überwindung menschlicher und weltlicher Bedingtheit, ist diese Kunst weder Idolatrie noch Gottes-Kunst, sondern list im doppelten Sinn: Handwerks- und Technikgeschick wie aber auch Täuschungs-Geschick. ${ }^{11}$

In der Konsequenz dieses hier kurz skizzierten doppelten Kunstverständnisses in der christlichen Tradition, das das (un)heilwirkende Bild als Heils- oder Teufelsbild von der technisch-magischen Könnerschaft einer Beherrschung von Materie und Naturgesetzen, aber auch einer artistischen Brillanz und Illusionsproduktion absetzt, sind die in der mittelalterlichen Literatur beschriebenen großen Kunstwerke alle im außerchristlichen, im heidnischen Bereich zu finden (durch die Herkunft der Künstler oder den Standort). Die Perfektion der in diesen Kunstwerken angestrebten Wahrnehmungstäuschung ist die Belebung des Kunstwerks, sei es mit mechanischen oder illusionistischen Mitteln (,als wenn es leben würde“"). ${ }^{12}$ Kunstwerke im christlichen Kontext dagegen werden nie in ihrer Entstehung beschrieben, sondern nur in ihrem Wirkungszusammenhang. Und hier dann ad bonam und ad malam partem, als heilwirkende, wunderwirkende Bilder oder als Teufelsinstrumente und Mittel teuflischer Irreführung. ${ }^{13}$ Wenn hier beschrieben wird, bezieht es sich auf die Rahmung eines Heilsgegenstandes, die allein zur Einbindung des immateriellen Werts in einen materiellen Wertkontext dient, ohne das Eingefasste $\mathrm{zu}$ berühren. Die dafür benötigten Materialien entstammen in der Regel der Fremde, der nicht-christlichen Welt, sind eine Art spolienhaft instrumentalisierte fremde

telalterliche Automatisierung im Spannungsfeld politischer, theologischer und technologischer Naturkonzepte. In: Automaten in Kunst und Literatur des Mittelalters und der Frühen Neuzeit. Hrsg. von Klaus Grubmüller, Markus Stock. Wiesbaden 2003 (Wolfenbütteler Mittelalter-Studien. 17), S. 91-117, hier S. 96. Die Verbindung von Zoroaster und Magie in der christlichen Tradition geht wohl auf die Ps.-Clementinen zurück.

11 Zur Semantik von ,machina', die noch die Bedeutung von ,Trick', ,List', ,Manipulation gegen die Natur' bewahrt vgl. Friedrich (Anm. 10), S. 97. Zu einer differenzierten Darstellung des intrikaten und engen Verhältnisses von Mechanik/Technik und Magie vgl. den reichen Aufsatz von William Eamon: Technology as Magic in the Late Middle Ages and the Renaissance. In: Janus 70 (1983), S. 171-212. Zu differenzieren ist aber zwischen einer auf Nutzen hin angelegten Mechanik und einer auf Unterhaltung, Repräsentation angelegten Mechanik und Technik, die stärker innerhalb eines „Kunstdiskurses“ (avant la lettre) mit Magie verbunden werden.

12 Dazu Klaus Niehr: ad vivum - al vif. Begriffs- und kunstgeschichtliche Anmerkungen zur Auseinandersetzung mit der Natur in Mittelalter und früher Neuzeit. In: Natur im Mittelalter. Konzeptionen - Erfahrungen - Wirkungen. Akten des 9. Symposiums des Medivistenverbandes, Marburg 14.-17. März 2001. Hrsg. von Peter Dilg. Berlin 2003, S. 472-487. Zu den Automaten als Illusionspoduktoren vgl. auch Friedrich (Anm. 10), S. 91. Dies gehört in den Bereich eines schon in der Antike unzählige Anekdoten hervorrufenden Kunstverständnisses, dessen Grenze die Lebendigkeit ist und dessen Ziel es ist, diese Grenze zumindest im ,als wenn“ zu überschreiten. Vgl. dazu: Ernst Kris, Otto Kurz: Die Legende vom Künstler. Ein geschichtlicher Versuch. Frankfurt a. Main 1995, S. 89-99; Eamon (Anm. 11), S. 174.

13 Ausführlicher zu den hier sehr kurz skizzierten zwei Kunstkonzepten: Schnyder (Anm. 3). 
Kunst. ${ }^{14}$ Sehr deutlich wird dies in der Gralsszene in Wolframs von Eschenbach „Parzival“, die in ihrem unglaublichen Pomp als Rahmung des Grals gelesen werden kann, der seinerseits sowohl in seinem Aussehen wie seiner Herkunft eine Leerstelle in der fremden Exotik bleibt. ${ }^{15}$ Die kostbaren Gegenstände und Materialien, die rundherum aufgefahren werden, haben alle eine fremde, heidnische Herkunft. Handwerker oder Künstler gibt es in der Gralswelt selber nicht, genauso wie die Gralswelt bildlos ist und der Gral nur in seiner heilsbringenden Wirkung Thema wird. ${ }^{16}$

Wenn in der christlichen Welt ein kostbar und teuer gefertigtes Kunstwerk Thema wird, gilt es auch immer gleich das Materielle durch eine Bedeutungsstruktur zu überhöhen und zu negieren. Sehr deutlich wird das in den Baukonzeptionen für Cluny und den diese begleitenden Schriften des Abtes Suger. Einerseits werden hier der unglaubliche ökonomische Aufwand wie auch die materielle Kostbarkeit des Baus herausgestrichen, gleichzeitig aber auch immer sofort die Materialität in die Spiritualität, in die Bedeutung hin aufgelöst. Dies geschieht durch Bildprogramme (das Leben Jesu auf den Haupttüren) und/oder Inschriften, wie zum Beispiel auf dem Antependium, dessen Inschrift in den zwei Versen gipfelt: Significata magis significante placent. ${ }^{17}$ Der abundante ökonomische Aufwand wird zum Rahmen und materiellen Anlass einer unbezahlbaren Wahrheitserkenntnis. Die Kostbarkeit ist einerseits einzig adäquater Hinweis auf den unsichtbaren und unfassbaren Wert, der darüber erschlossen werden kann, wird gleichzeitig aber auch gerade durch den so erschlossenen Wert depotenziert und zunichte gemacht. Die Darstellung fremder Kunstwerke dagegen, heidnische Heiligtümer und Prachtbauten mit ihrer kunstvollen Ausstattung, bleibt in der materiellen Kostbarkeit und deren Glanz stecken, ohne Deutungspotenzial auf einen geistigen Sinn hin.

Schematisierend kann man in Bezug auf die literarische Kunst-Darstellung von einer Rhetorik der christlichen Kunst sprechen, die eine Rhetorik der Entmaterialisierung, der Durchsichtigkeit, der Ursprungslosigkeit und der offenbarenden Authentizität ist; in Bezug auf die Darstellung nicht-christlicher Kunst aber von einer Rhetorik der Materialisierung, der Gemachtheit, der Kunstfertigkeit, der Technik und der täuschenden Raffinesse. $^{18}$

14 Vgl. zu der Funktion der umhüllenden Materialität auch Bynum (Anm. 4), S. 58 u. ö. Zu denken ist in diesem Kontext auch an die Reliquienschreine.

15 Wolfram von Eschenbach: Parzival. Nach der Ausgabe Karl Lachmanns revidiert und kommentiert von Eberhard Nellmann, übertragen von Dieter Kühn. Frankfurt a. Main 1994 (Bibliothek des Mittelalters. 8,1-2).

16 Vgl. dazu auch: Mireille Schnyder: Überlegungen zu einer Poetik des Staunens im Mittelalter. In: Wie gebannt. Ästhetische Verfahren der affektiven Bindung. Hrsg. von Martin Baisch, Andreas Degen, Jana Lüdke. Freiburg 2013, S. 95-113.

17 Andreas Speer, Günther Binding (Hrsg.): Abt Suger von Saint-Denis. Ausgewählte Schriften: Ordinatio, De consecratione, De administratione. Darmstadt 2008, S. 323-325 u. S. 330 f.

18 Wenn Bynum für das Spätmittelalter eine Fokussierung und Ausstellung der Materialität der Bilder konstatiert, so im Kontext einer Brechung jeder Illusionierung, einer Negation dessen, was das heidnische Bild als belebtes Bild auszeichnet. Und auch da ist nicht die Herstellung, die Kunstfer- 


\section{Meisterliche (Wort)Kunst}

Was in der mittelalterlichen Literatur auf der Ebene der Beschreibung von Kunstwerken sehr deutlich als Differenz der Kunstkonzeption bezüglich christlicher oder heidnischer Kultur und damit als Differenz von christlicher und heidnischer Kunst gesehen werden kann, hat auch Implikationen für die Poetik oder besser: die Konzeptualisierung des Dichtens. Denn die Entstehung und raffinierte Gemachtheit der fremden Kunstwerke (Statuen, Bauten, Grabmäler, [Wand-]Bildprogramme, Brunnen, Räume, Betten, etc.) wird nicht nur detailliert beschrieben, sondern oft auch eng an das Erzählen zurückgebunden und so zu einem Modell dichterischen Schaffens. Die beschriebenen Werke, meisterlîch geworht und meisterlîch gezieret, sind immer auch Teil einer Texttradition und damit (auch) Werke eines Erzählers. ${ }^{19}$ So wendet sich zum Beispiel der Erzähler im „Herzog Ernst“, nach der üppigen Beschreibung des blendend schön gebauten und geschmückten Grippia und dem Hinweis, dass wir das alles aus Büchern wissen, dâ ez an geschriben stât (V. 2245), an das Publikum mit dem Wunsch: wol im derz uns getihtet hât | sô rehte wol ze tiute (V. 2245 f.: „Möge es dem wohl ergehen, der es für uns geschaffen hat, so richtig gut zu verstehen."). In dieser Engführung von Ekphrasis und Bauwerk wird das letztere zu Text und darüber zum Objekt der Auslegung, der Deutung, der Exegese, aber auch der Übertragung in die deutsche Sprache, den eigenen Verstehensraum. ${ }^{20}$

Damit schließt der Dichter sein Erzählen bewusst an das Kunstwerk der „Fremden“ an und stellt damit implizit seine Kunstfähigkeit, seine Technikbeherrschung und seine auf Illusionierung angelegte Könnerschaft aus. Die beschriebene meisterschefte ist seine eigene. Es ist nicht der von Gott inspirierte Dichter, der sich hier inszeniert, sondern der Könner, der sich der Wirkmacht illusionistischer Effekte bewusst ist und deren Techniken beherrscht. Gleichzeitig ist es das in den Kontext des Erzählkunstwerks, in die eigene Sprache gebrachte fremde Bauwerk, das ze tiute vorgelegt wird. Damit wird die Kunst der Materialbeherrschung über die Kunst der Wortbeherrschung in einen Kontext der Sinnhaftigkeit und Sinngenerierung hereingeholt, der das fremde Kunstwerk deutbar macht. ${ }^{21}$

tigkeit im Blick, sondern die Materialität des Kunstprodukts, die Entlarvung des Scheins auf ein im Material Bedeutetes hin. Bynum (Anm. 4), S. 38: „Indeed, it is a characteristic of medieval images that their crafters tended to employ materials explicitly as themselves rather than creating an illusion, or a naturalistic depiction, of them through other media."

19 Zitate aus der Beschreibung von Grippia in: Herzog Ernst. Ein mittelalterliches Abenteuerbuch. In der mittelhochdeutschen Fassung B nach der Ausgabe von Karl Bartsch mit den Bruchstücken der Fassung A. Hrsg. von Bernd Sowinski. Stuttgart 1979, V. 2239 u. V. 2235.

${ }^{20}$ V. 2246 f. kann auch auf den am Ende der Überlieferungsreihe deutsch erzählenden Autor bezogen werden.

${ }^{21}$ In Bezug auf den Herzog Ernst sind es über das Narrativ konstituierte Deutungen im heilsgeschichtlichen Rahmen einer Versuchungslogik und Scheinhaftigkeit des Teuflisch-Bösen. 
Anders als die Machart und Technik fremder Kunstwerke ist die Machart christlicher Kunstgegenstände dagegen in den Texten nicht Vergleichsmoment für das Dichten. Hier wird kein Mechanismus beschrieben, keine technische Raffinesse entlarvt, sondern nur verschleiert, ohne dass ein Kunst-Werk in den Blick käme: die Beschaffenheit des Grals ist unvorstellbar. ${ }^{22}$ Dafür aber schließt sich die dichterische Rede an die Anfangslosigkeit und Ungemachtheit dieser Gegenstände an und stellt sich als Mittel einer wahren Rede dar. Die Setzung eines transzendenten Ursprungs legitimiert und valorisiert das dinc, aber auch den im Text geschlossenen Glaubenspakt zwischen Erzähler und Rezipient, der jeden Zweifel an der Heils- und Wirkmacht des Grals und deren Darstellung ausschließt. ${ }^{23}$

Was in der Kunst-Beschreibung innerhalb der Texte wirksam ist, gilt auch für die Inszenierung der Textproduktion selber. Ein geistlicher Text ist ein non manu factum, eine nur durch göttliche Inspiration ermöglichte Dichtung, deren Erzähler Sprachrohr Gottes ist. Anders die weltliche Dichtung, die sich in eine innerweltliche Texttradition stellt, die in der Regel in die heidnische Zeit zurückgeht, in ihrer christlichen Lesart dann aber zum eigentlichen Sinn findet.

\section{Übertragungen I}

Die der heidnischen Fremde zugschriebene Kunstfertigkeit als Vergleichsmoment für die dichterische Fähigkeit der Illusionierung und deren Techniken wird in der zweiten Hälfte des 13. Jahrhunderts vermehrt in den eigenen Handlungs- und Lebensbereich hereingeholt. Es ist nicht mehr nur die Anbindung der eigenen Wortkunst an die heidnische Beherrschung der Künste und Verfügung über materielle Kostbarkeit, wie in den als Kunstwerke des Dichters inszenierten fremden Kunstgegenständen innerhalb des Narrativs, sondern die Wortkunst selber wird Thema und als mit kostbarsten Materialien und größtem Geschick arbeitendes Handwerk dargestellt. ${ }^{24}$ Wird die exquisite Kostbarkeit heidnischer Kunstwerke durch die Einbindung in die sprachliche Darstellung in den christlichen Deutungsrahmen hereingeholt, indem der Schein mit Sinn aufgeladen zum Zeichen wird, wird nun vermehrt die (christliche) Wortkunst, als rare Kunst, die mit kostbarsten Materialien in größter Raffinesse umgeht, in den Kontext fremder, auch magisch konnotierter Objekte gestellt. Dabei ist es gerade die Exquisität der Dichtkunst, ihre Seltenheit, die das Dichtwerk zu etwas Exotischem, Fremdem, Seltenem und Kostbarem macht, das sich mit raren Edelsteinen oder auch dem Phönix

22 Vgl. auch Schnyder (Anm. 16).

23 Vgl. Wolfram von Eschenbach: Parzival (Anm. 15), V. 238,8-17. Genauer dazu: Schnyder (Anm. 16), S. 107-111.

${ }^{24} \mathrm{Zu}$ dem in der Zeit verstärkt auftretenden Bildfeld des Handwerks für das Dichten vgl. Sabine Obermeier: Von Nachtigallen und Handwerkern. ,Dichtung über Dichtung ' in Minnesang und Sangspruchdichtung. Tübingen 1995 (Hermaea NF 75), S. 333-338. 
vergleichen lässt, wie es Konrad von Würzburg im Prolog zum „Trojanischen Krieg“ sagt.
sît man gimmen reine
dar umb ie künde triuten,
daz si niht al den liuten
wol veile sint, sô diuhte mich
gevellic unde mügelich,
daz guot getihte ware
ze hove niht unmare
durch sîne tiuren fremdekeit.
diu schrift von einem vogele seit,
der fênix ist genennet.
[...]
ich wil den spahen orden
getihtes ime gelîchen. (V. 24-47)

Nachdem man seit jeher schöne Edelsteine deshalb wert schätzt, weil sie nicht allen Menschen wohlfeil sind, so scheint es mir recht und billig, dass ein gutes Gedicht am Hof nicht missachtet würde, wegen seiner wertvollen Seltenheit. Die Schrift berichtet von einem Vogel, der Phönix heißt. [...] Den exklusiven Stand der Dichtung will ich mit ihm vergleichen.

Auffallend bei diesem Vergleich ist jedoch, dass die (gute) Dichtung nicht mit fremden Kunstwerken, mit raffiniert bearbeiteten kostbaren Materialien verglichen wird, sondern mit Schöpfungswundern, deren seltene Schönheit und wunderbare Kräfte letztlich auf Gott zurückgehen. Damit wird das Dichten zu einem Wunderwirken Gottes, gehört die Dichtkunst zu den mirabilia mundi. Das Verständnis der (weltlichen) Dichtkunst, als Sprachkunst, lehnt sich so trotz allem auch an die das christliche Bildverständnis prägende Tradition des nicht von Menschenhand gemachten Kunstwerks an. Entsprechend ist das $\mathrm{A}$ und $\mathrm{O}$ des Dichters die Herzenseinsicht, daz sîne fuoge und sîne kunst I nâch volleclichen êren I mac nieman in gelêren, I wan gotes gunst aleine (,dass seine Geschicklichkeit und sein Können zu vollkommenem Ansehen niemand ihn lehren kann außer Gottes Gunst allein). ${ }^{25}$

Der Spruch 32,301 von Konrad von Würzburg formuliert diese Idee einer absoluten Kunst der Dichtung aus: ${ }^{26}$

Für alle fuoge ist edel sang getiuret und gehêret,

darumbe daz er sich von nihte breitet unde mêret.

elliu kunst gelêret

mac werden schône mit vernunst,

wan daz nieman gelernen kan red und gedœne singen;

${ }^{25}$ Konrad von Würzburg: Der Trojanischer Krieg. Nach den Vorarbeiten K. Frommanns und F. Roths hrsg. von Aadalbert von Keller. Stuttgart 1858 (Bibliothek des literarischen Vereins in Stuttgart. 44), V. 72-77.

${ }^{26}$ Konrad von Würzburg: Kleinere Dichtungen III, Die Klage der Kunst, Leiche, Lieder und Sprüche. Hrsg. von Edward Schröder, mit einem Nachwort von Ludwig Wolf. 4. Aufl. Dublin/Zürich 1970, S. 66. 
diu beidiu müezen von in selben wahsen unde entspringen:

$\hat{u} z$ dem herzen clingen

muоz ir begin von gotes gunst.

ander fuoge durfen alle râtes und geziuges wol.

swer si trîben rehte sol,

der muoz hân daz gerüste,

dâmite er si volende nâch der liute muotgelüste;

son darf der sanc niht helfe wan der zungen und der brüste:

sunder valsche âküste

gât er dâvon für alle kunst.

Vor allen Kunstfertigkeiten ist der edle Gesang geliebt und verehrt, weil er sich aus Nichts ausbreitet und mehrt. Alle erlernte/gelehrte Kunst kann mit Hilfe von Verstand schön werden, nur Rede und Töne-Singen kann niemand lernen; diese beiden müssen aus sich selber wachsen und entstehen: Aus dem Herzen klingen muss ihr Anfang durch die Gnade Gottes. Andere Künste brauchen alle Unterweisung und Gerätschaften. Wer auch immer sie richtig betreiben will, der braucht die Ausrüstung, damit er sie nach der Leute Verlangen ausführen kann. Der Gesang aber braucht keine Hilfsmittel außer der Zunge und der Brust: Ohne falsche Tücke geht er deshalb aller Kunst vor.

Konrad greift das christlich-religiös aufgeladene Kunstverständnis auf, das in der Ungemachtheit, der Materialitätslosigkeit, der Unabhängigkeit von menschlich erlernbaren Techniken und damit der Unverfälschtheit einer göttlich inspirierten Aussage sein Ideal hat. ${ }^{27}$ Während sich jede andere Kunst nâch der liute muotgelüste $(32,312)$ richte, also von Willkür und Rezipientenwünschen abhängig sei, brauche der Gesang nichts als Zunge und Brust und ist so frei von falscher Absicht, ohne Tücke und ohne Entstellung. In der Wortwahl geht Konrad sogar so weit, über die ersten sieben Verse eine Parallelisierung zur Schöpfung ex nihilo (von nihte/von in selben) zu evozieren, die dann genau in der Mitte des Spruchs ihre Differenz zur göttlichen Schöpfung in der Bestimmung der bewegenden Kraft im Anfang findet: gotes gunst. Damit werden Anfang und Produktionsprozess der Dichtung verschleiert. Die Logik der Unterscheidung von Dichtung und anderen Künsten ist dieselbe Logik, die im christlichen Kunstdiskurs das christliche Kultbild von dem heidnischen Kunstbild unterscheidet. Entsprechend ist diese Dichtungstheorie auch wieder eine Rezeptionstheorie: Gute Dichtung zu bestimmen ist Aufgabe der Rezipienten. Diese können entweder mit hellem Geist die wahre Dichtung erkennen, oder mit der Blindheit der Fledermäuse das faulende Holz für das wahre Licht nehmen, wie es im Prolog zum Trojanischen Krieg heißt (Tr.Kr. V. 154-169).

Was im allgemein formulierenden Spruch zum inspirierten, gott-gemachten Werk wird, ist dann aber im Moment, wo ein sprechendes Ich ins Spiel kommt, in den Raum der menschlichen Fähigkeiten, der Willkür, der Verpflichtungen und Arbeitsmühen geholt. Mit dem Auftreten eines Erzähler-Ich tritt die göttliche Inspiration zurück zugunsten der Vernetzungen des Ich in soziale und ökonomische Zusammenhänge und der Betonung seiner Abhängigkeit von physischen und intellektuellen Fähigkeiten

27 Zur Tradition dieses Dichtungsverständnisses vgl. auch Pékary (Anm. 7). 
(Tr.Kr. V. 176-269). Gleichzeitig tritt die Kunstfähigkeit, das Können dieses Ich in den Vordergrund und wird der Arbeitsprozess Thema, die Suche nach dem Anfang der Erzählung, den Textquellen, den richtigen Worten. Und die in der Dichtungs-Theorie des Spruchs ausgeschlossenen muotgelüste sind hier durch die Nennung des Auftraggebers wieder eingeholt: daz ich ez hebe mit willen an, I dar ûf hât wol gestiuret mich | der werde singer Dietrich I von Basel an dem Orte (Tr.Kr. V. 244-247).

Das Dichtungsverständnis changiert so zwischen der abstrakten Idee (göttlich) inspirierter Rede und dem Konzept artistisch-raffinierter Könnerschaft im Dienste weltlicher Interessen, zwischen christlicher Inspiration und heidnischer Kunstbeherrschung (Meisterschaft), göttlicher Wirkung und magischen Techniken, Gottwirken und Kunstkönnen.

Dem Spruch 32,301 geht ein Spruch im gleichen Ton voran, ${ }^{28}$ in dem der Gesang des Meissners vorgestellt wird als eine Kunst, die dieser während eines Greifenflugs von Sirenen gelernt hätte und sie nun im Schrein seines Herzens bewahre, um damit alle rheinländischen Sänger zu übertrumpfen. Diese nicht inspirierte, sondern erlernbare, wenn auch in einem mythisch überhöhten Raum erlernbare Kunst, ist jedoch reine Unterhaltungskunst, eng verbunden mit der Heldenepik. ${ }^{29}$

Der Mîssenaer hât sanges hort in sînes herzen schrîne,

sîn dôn ob allen razen doenen vert in êren schîne,

dâmit er bî Rîne

die singer leit in sîn getwanc.

in fuorten überz lebermer der wilden grîfen zwêne:

dâ lêrte in underwegen doene singen ein syrêne:

lebte noch Elêne

von Kriechen, si seit im ir danc

durch sîn adellichez donen, daz dâ klinget hôhe enbor.

er gât an der wirde vor

smaragden und saphîren;

er doenet vor uns allen sam diu nahtegal vor gîren;

man sol ze sînem sange ûf einem messetage vîren.

,alsus kan ich lîren',

sprach einer der von Ecken sanc. (32,286-300)

Der Meissner hat einen Liederschatz im Schrein seines Herzens, seine Weise fliegt über allen scharfen Tönen im Schein der Ehre, damit er am Rhein die Sänger in seinen Zwang nimmt. Zwei wilde Greife führten ihn übers Lebermeer: Unterwegs lehrte ihn da eine Syrene Melodien singen. Wenn Helena aus Griechenland noch leben würde, sie würde ihm danken für sein edles Singen, das da hoch hinauf klingt. Er übertrifft an Wert Smaragde und Saphire, er klingt vor uns allen wie die Nachtigall vor Geiern; man soll zu seinem Sang auf einem Jahrmarkt feiern. ,Genau so kann ich leiern', sagte einer, der von Ecke sang.

${ }^{28}$ Konrad von Würzburg (Anm. 25), S. 65.

29 Zur pejorativen Deutung dieser Stelle in Bezug auf die Heldenepik als Unterhaltungsliteratur vgl. Rüdiger Brandt: Konrad von Würzburg. Kleinere epische Werke. Berlin 2000 (Klassiker Lektüren. 2), S. 60. 
Die Differenz zwischen christlich-inspirierter Kunst als Gottes-Kunst in Spr 32,301 und magisch-heidnischer Kunst als erlernbarem Können, das hier mit mythisch-magischen Mitteln arbeitet, ist deutlich bezeichnet. Ist die eine dank göttlicher Gnade ein HerzensKlang, ist die andere, dank mythisch-magischem Wissen ein Herzens-Schatz, von einer Kostbarkeit (wirde), die jeden Edelstein in den Schatten stellt. Aber die Sirenenklänge dieser Dichtung sind verführerisch, ihre Weise (dôn) erhebt sich im Glanz der Ehre (êren schîne) über alle scharfen (razzen) Klänge, wodurch die Sänger am Rhein überwältigt werden. Doch gehört diese Art des Singens vor ein Jahrmarkt-Publikum.

Auffallend ist, dass Konrad die zwei Sprüche mit einer konträren Medialität ausstattet. Ist das Lob der idealen Dichtung in 32,301 ein Lob des Klangs, ist die kritisierte magisch-verführerische Kunst in 32,286 über ihren Glanz und ihren Smaragde wie Saphire übertreffenden Schatz im Schrein des Herzens vor Augen gestellt. Damit ist die göttlich inspirierte Dichtung Klang-Kunst, die magisch-mythisch wirkende Dichtung aber ein in exotischen Gegenden gelerntes, verführerisches, glänzendes Singen, das nicht nur magisch bezwingt, sondern auch als Schatz im Herzen gehortet werden kann. Ist das erste ursprungsloses, göttlich initiiertes Klingen, ist das andere magisch wirkendes, kostbares Wissen, wie es der schönen Helena - als Inbegriff fataler Schönheit gefallen hätte und wie es die ungebildete Masse blendet. ${ }^{30}$

\section{Übertragungen II}

Im Kontext dieses Konrad'schen Verständnisses einer wahren Dichtung, in dem das Herz Quell eines von Gott initiierten Singens ist, während jede auf blendende und verführerische Tricks setzende Dichtung zwar die Kostbarkeit von Edelsteinen übersteigen mag, in der Wirkung aber fatal ist, ist es nun doch erstaunlich, wenn Konrad sein großes Marienlob mit dem Wunsch beginnt, in seiner Herzensschmiede ein Gedicht für Maria aus Gold herzustellen: ${ }^{31}$

Ei künde ich wol enmitten

in mines herzen smitten

getihte uz golde smelzen,

und liehten sin gevelzen

von karfunkel schone drin

30 Zur Bedeutung des Klangs für Konrads Poetik vgl. die präzisen, bis in die feinsten Strukturen des Textes eindringenden Analysen von Susanne Köbele: Zwischen Klang und Sinn. Das GottfriedIdiom in Konrads von Würzburg Goldener Schmiede (mit Anmerkungen zur paradoxen Dynamik von Alteritätsschüben). In: Alterität als Leitkonzept für historisches Interpretieren. Hrsg. von Anja Becker, Jan Mohr. Berlin 2012 (Deutsche Literatur. Studien und Quellen. 8), S. 303-333, hier S. $310 \mathrm{ff}$.

31 Konrad von Würzburg: Die goldene Schmiede. Hrsg. von Edward Schröder. 2. Aufl. Darmstadt 1969. Die angekündigte historisch-kritische Ausgabe von Karl Bertau ist mir leider noch nicht zugänglich. 
dir, hohiu himelkeiserin!

so wolte ich diner wirde ganz.

ein lop durliuhtec unde glanz

daruz vil harte gerne smiden. (V. 1-6)

Ach könnte ich doch mitten in meiner Herzensschmiede Dichtung aus Gold schmelzen und hellen Karfunkel-Sinn einlassen für dich, hohe Himmelskaiserin! So würde ich zu deiner vollkommenen Ehre sehr gern ein durch und durch leuchtendes und glänzendes Lob daraus schmieden.

Gleich am Anfang des Textes wird der Blick in die Herzkammer gelenkt, in der sich eine Goldschmiedewerkstatt installiert, in der das Marienlob geschaffen werden soll. Damit kommt da, wo sonst gerade die Kunstlosigkeit des Inspirationsvorgangs als Verschleierung des Herstellungsprozesses inszeniert wird, der Dichtungsprozess als Handwerk in den Blick. Gleichzeitig stellt sich das Ich ins Zentrum des Schaffensprozesses. Das Herz, Ort göttlicher Inspiration, wird - im idealen Wunsch - zur Werkstatt, in der das Ich mit wertvollen Materialien zu Gange ist. Das hier so prominent auftretende Ich ist dieses Ich, das sich auch in den Prologen der weltlichen Werke jeweils sozusagen im Rücken der idealen Konzeption der Dichtkunst als Werk göttlicher Inspiration an die mühevolle, in weltlichen Netzen verstrickte Arbeit macht. Anders als dort steht es hier aber am Anfang und stellt sich - sowohl im Text wie im Bild - in den Raum, der eigentlich der göttlichen Inspiration vorbehalten ist. Und es ist kein Klingen, das hier aus dem Herzen fließt, sondern nur der leise Klang der Werkzeuge, die das Gold und die Edelsteine zu einem leuchtenden, glänzenden Kunstwerk als Lob Mariens verarbeiten sollen. Nicht von nihte, sondern mit Hilfe von Materialien bester Qualität wird hier gearbeitet. Die dafür notwendigen Werkzeuge sind zwar - wie in der topischen $\mathrm{Ab}$ grenzung von den Instrumenten der anderen Künste - nur Zunge und Mund, doch wird über die Metaphorik die Distinktion unterwandert: Hier wird gehämmert und gereinigt. Und es ist gerade der meisterhafte Umgang mit diesen Werkzeugen, woran die Defizienz des Ich deutlich wird: Es ist zu wenig geschickt für seine Aufgabe. So bereiten ihm die Gliederwerkzeuge Zungenhammer und Mund Schwierigkeiten:

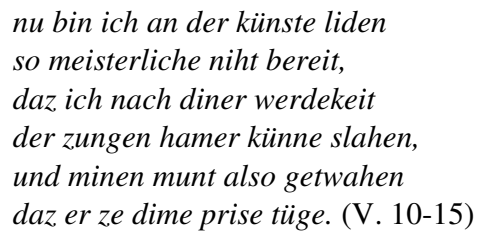

Nun bin ich in Bezug auf die Glieder der Künste nicht so meisterlich ausgestattet, dass ich deiner Würde gemäß den Zungenhammer schlagen und meinen Mund so reinigen könnte, dass er zu deinem Preis taugen würde.

Hier interessiert nun nicht die Unsagbarkeits- und Unfähigkeitstopik, sondern die Inszenierung der Dichtung als Kunst-Werk am Anfang eines Marienlobs, das im Kontext der Tradition geistlicher Dichtungen eine initiale Inspirationsbitte erwarten ließe. Wenn hier nun der Schaffensprozess thematisiert wird als ein Arbeitsprozess, der Kunstfertig- 
keit verlangt, stellt sich dieser Anfang in direkten Gegensatz zu der die Kunst religiös legitimierenden Tradition der Verschleierung der Künstlerhand. Dass das Kunstwerk als Menschenwerk deutlich gemacht wird, kann im Kontext der von Bynum betonten Aufmerksamkeit auf die Materialität im späteren Mittelalter gesehen werden. ${ }^{32}$ Anders als in den von ihr vorgestellten Beispielen aber geht es hier nicht nur darum, das Material als Hülle, als Anlass oder als Mittel der Wahrnehmung von Transzendenz zu erkennen, sondern in der Beobachtung des Herstellungsprozesses geht es um die Einbindung des Künstler-Ichs in das Werk. In diesem Ich ist der Ursprung von Wille, Konzept und Ausführung des kostbaren Kunstwerks zu finden. Was mit den heidnischen Kunstgegenständen immer schon in Verbindung gebracht war, Geschicklichkeit, exklusives Wissen und technische Fähigkeit ihrer Hersteller als Mittel der Transformation der Materie in einen Gegenstand, der Verwunderung und Bewunderung hervorruft, als Mittel aber auch der Transgression der Materie in illusionistischen Schein, wird hier - im Marienlob - für christliches Kunstschaffen reklamiert. Damit wird aber auch die Grenze zwischen christlich-religiös legitimierter Kunst als Wahrheitsvermittlerin und nur im heidnisch-fremden Raum sich legitimierender Kunst der Illusionierung, technischen Manipulation und Faszination durchlässig.

Dass das Werk Konrads dann an der Aufgabe einer Darstellung von Mariens unbilde (V. 566) scheitert, ja scheitern muss, unterstreicht gerade seinen Ursprung im menschlichen Ich, das mit den ihm gegebenen Mitteln und Fähigkeiten auskommen muss und deshalb immer im Wunsch, im Nicht-Erreichten bleibt. Es ist nicht die Gnade Gottes, die ihm die Möglichkeit des Dichtens gibt, sondern die Güte Mariens, die das in der Ausführung nicht den Willen einholende Kunstwerk als Marienlob akzeptiert. ${ }^{33}$

Die Kunsthandwerksmetaphorik des Marienlobs schreibt dem Künstler im geistlichen Kontext eine Präsenz und Beteiligung am Werk zu, die zuvor nur im weltlichen (wenn nicht heidnischen) Kontext beobachtet werden kann. Entsprechend führt dies implizit zu einer „Säkularisierung“ des geistlichen Dichtens. Wenn de Boor in Bezug auf Konrad von einem „kunsthandwerklich bestimmten Selbstgefühl“ spricht, trifft das in einem heutigen Verstehenshorizont von „kunsthandwerklich“ nur die eine Seite. ${ }^{34}$ Denn „kunsthandwerklich“ im Kontext dieses dichterischen Schaffens meint auch ein Kunstkonzept, das das Ich des Dichters aus seiner Mittlerrolle zwischen Gott und Welt befreit und in den Bereich innerweltlicher Kunst stellt, die menschlichem Wünschen und Wollen Form gibt. ${ }^{35}$

32 Vgl. Bynum (Anm. 4).

33 also daz mir Cuonrade I von Wirzeburc daz heil geschehe, I daz mir din güete übersehe I swaz ich vermide an dinem lobe. Die goldene Schmiede (Anm. 30), V. 120-123.

34 Helmut de Boor, Richard Newald: Geschichte der deutschen Literatur Bd. 3/1: Die deutsche Literatur im späten Mittelalter 1250-1350. Neuberarbeitet von Johannes Janota. München ${ }^{5} 1997$, S. 46.

35 Die Überlegungen lassen sich bis zu einem gewissen Grad an Stierles These einer neuen „Idee des Kunstwerks als Ort der Überschreitung der Faktizität aber zugleich der in keiner Dienstbarkeit mehr aufgehenden Selbstbezüglichkeit" anschließen, wobei ich den von Stierle gebrauchten Begriff einer ,,immanenten Transzendenz“ nicht aufnehmen wollte, da er die kategoriale Differenz einer re- 
Wenn dann - in der Fortsetzung des hier beobachteten Wandels im Dichtungsverständnis - in einem Spruch des Langen Tons von Frauenlob $(\mathrm{V}, 13)$ das Dichten selber mit der Arbeit eines Baumeisters gleichgesetzt wird, ist die Technik und Kunstfertigkeit des Dichters, seine Könnerschaft als menschliche Könnerschaft, ganz aus dem Horizont der Inspirationslehre gelöst, dafür - als Fürstenlob - in den Kontext repräsentativer Architektur gestellt. Dabei fällt auf, dass auch hier prominent ein Ich sich in (die weltliche) Szene setzt: ${ }^{36}$

$\mathrm{J}$ a tun ich als ein wercman, der sin winkelmaz

ane unterlaz.

$z u$ sinen werken richtet,

[ ] uz der fuge tichtet

die höhe und lenge: wit und breit, alse ist ez geschichtet;

und swenne er hat daz winkelrecht nach sinem willen gezirket,

Darnach er danne wirket, als man wirken kan.

nu merket an,

ich forme, ich model, ich mizze:

wie gerne ich mich flizze

eins lobes, daz hat so hoch ein name, daz ich sin nicht vergizze!

ez. höhet, lenget, breitet sich, sin nennen niendert lirket.

Ez ist gekrönet, guldin, glanz,

gespiegelt, luter, sunder schranz,

materjen ganz,

milte als ein cranz.

ez, zafet, zieret sinen swanz:

froun Eren diener Vivianz

ist Waldemar, der fürste stolz, $\quad$ sin lob noch wunder wirket.

Ja, ich mache es wie ein Baumeister, der sein Winkelmaß ständig an seine Werke anlegt, [ ] aus der Fuge heraus die Höhe und Länge entwirft: Weit und breit, so ist es angelegt. Und wenn er den rechten Winkel nach seinem Willen abgemessen hat, schafft er, wie man schaffen kann. Nun passt auf: Ich forme, ich modelliere, ich vermesse: Wie gern befleißige ich mich eines Lobes,

ligiösen Transzendenz und der dichterischen/künstlerischen Überschreitung des Realen nivelliert. Karlheinz Stierle: Säkularisierung und Ästhetisierung in Mittelalter und Früher Neuzeit. In: Ästhetik, Religion, Säkularisierung I. Von der Renaissance zur Romantik. Hrsg. von Silvio Vietta, Herbert Uerlings. München 2008, S. 55-74, Zitat S. 57.

36 Frauenlob (Heinrich von Meissen). Leichs, Sangsprüche, Lieder. 1. Teil. Einleitung, Texte. Auf Grund der Vorarbeiten von Helmuth Thomas hrsg. von Karl Stackmann, Karl Bertau. Göttingen 1981, S. 396. Dabei wird mit dem Baumeisterbild eine prominente poetologische Bildlichkeit aufgenommen, die die Dichtung als im Geist konzipiertes Werk thematisiert, das dann in der Ausführung erst ins Werk gesetzt wird. Vgl. dazu mit weiteren Verweisen: Obermeier (Anm. 24), S. 249 f. u. Anm. 342. Zur Tradition der im Bild des planenden Baumeisters implizierten Parallelisierung göttlicher und menschlicher Schöpfung vgl. Michael Stolz: ,Tum'-Studien. Zur dichterischen Gestaltung im Marienpreis Heinrichs von Mügeln. Tübingen/Basel 1996 (Bibliotheca Germanica. 36), S. 136-139, hier auch weitere Literatur. 
das einen so hohen Namen trägt, dass ich ihn nicht vergessen möge. Es höht, längt, breitet sich, sein Nennen stottert nie.

Es ist gekrönt, golden, glänzend, spiegelnd, rein, ohne Bruch, ein Stück. Freigebigkeit schmückt es wie ein Kranz, ziert seine Herrlichkeit: Der Ehrendiener Vivianz ist Waldemar, der stolze Fürst, sein Lob wirkt noch Wunder.

\section{Schluss}

Die grundsätzlich verschiedene Art der Kunstkonzeption und Kunstwahrnehmung, die sich in der christlich-theologischen Tradition zwischen dem Bild in christlichem Kontext und dem Bild in heidnischem Kontext installierte - und die in der literarischen Kunstdarstellung perpetuiert wurde -, wird im Blick auf Konrads Texte unfest. Und dies in doppelter Hinsicht: Einerseits wird Kunst (Dichtkunst) im allgemeinen Sinn (nicht nur im geistlichen Bereich) über eine in religiöser Tradition stehende Argumentation in einen die irdischen Bedingungen transzendierenden Kontext gestellt und damit der Künstler/Dichter implizit in einem Bezug zur Transzendenz (dem Sakralen) gesehen.

Anderseits wird durch die Fokussierung der Gemachtheit der Dichtkunst, des Entstehungsprozesses mit all seinen Wissen, Können und Geschick fordernden Schritten und dies im geistlichen Bereich -, die Gewalt des Einzelnen, der souverän und raffinert über die Materie und deren Kräfte verfügt, hervorgehoben.

In diesem Spiel zwischen Sakralisierung der Kunst und Säkularisierung der geistlichen Rede kann ein Reflex eines beginnenden Ablösungs- und AusdifferenzierungsProzesses des Kunst- und Dichtungsdiskurses aus dem religiös-theologischen Diskurs gesehen werden. Ob dies letztlich gelungen ist, kann man sich fragen.

Die Ansätze einer Ent-Auratisierung der Kunst, wie sie in der „Goldenden Schmiede“ durch Übertragung der Darstellungsart „heidnischer“ Kunst auf das geistliche Dichtwerk zu sehen sind, sind durch die Auratisierung der Kunst durch Übertragung des Konzepts sakraler Kunst auf die Kunst allgemein, verdrängt worden. Die bis in jüngere Zeit deutliche literaturgeschichtliche Vernachlässigung genau dieser ,entauratisierten" Texte, ${ }^{37}$ aber auch die kunstgeschichtliche Abwertung aller ausgestellten Kunstfertigkeit und Könnerschaft (man denke an bis heute nicht wertneutral verwendete Begriffe wie: Virtuosentum und Kunsthandwerk) ist nicht unbedingt Ausdruck eines säkularen Kunstverständnisses.

37 Für eine prägnante Zusammenfassung der Werturteile bezüglich der „Goldenen Schmiede“ Konrads vgl. Köbele (Anm. 30), S. 303-333, hier S. 305 ff. 
Bereitgestellt von | UZH Hauptbibliothek / Zentralbibliothek Zürich 